\title{
The Effect Factors of Projecting Velocity and Projecting Time of Video Scout Projectile
}

\author{
Li Yun ${ }^{\mathrm{a}}$, Zou Jun ${ }^{\mathrm{b}}$, Li Jun ${ }^{\mathrm{a}}$ \\ New Star Research Institute of Applied Technology, Hefei, 230031, China; \\ aemail:ljazq1314@163.com, bemail:2249861737@qq.com
}

Keywords: projectile rotational velocity, projecting charge, cabin opening altitude, projecting velocity, projecting time

\begin{abstract}
The paper, for the purpose of studying the relevant factors affecting the projecting velocity and time of video scout projectile, makes an analysis on the stress condition of projecting section of scout equipment, sets up the axial movement differential equation for scout equipment, and analyzes the influences of projectile rotational speed, projecting charge and cabin opening altitude on projecting velocity and time of video scout projectile. Analysis of this paper shows that when choosing the opening-point of video scout projectile, the downward arc stage is a correct choice, when designing the video scout projectile, the projecting charge can be determined the strength and reliability of projecting structure, and the changes in projecting height has little influences on projecting velocity and projecting time.
\end{abstract}

Video scout projectile is carried by shrapnel, rocket projectile and missile, and it is carried to the overhead space of target area and disperses the mission equipment from the projectile body by using the method of opening the cabin in the air, after which, the video scout equipments can stay in the air for a certain amount of time by using the method of rotary reduction and deceleration. During this process, the scout equipment timely transmit the signals it has gathered, which is to be further stored, processed and the target's coordinates can be defined in the ground system.

After projectile launching, the projectile is along the conventional uncontrollable trajectory and reaches the overhead space of the target area, and then the scout equipment is shot off from the projectile. The projecting process is crucial to the analysis of trajectory of the scout equipment, since it is the base for correctly analyzing two work stages: deceleration and despinning stage and steady falling stage, while the projecting velocity and time are two important parameters of projecting process. Therefore, by setting up the video scout projectile free-flight section model and scout equipment projecting section trajectory model, the paper analyzes the influences of projectile rotational speed, projecting charge and cabin opening altitude on projecting velocity and time.

\section{Building the Trajectory Model}

\section{The building of projectile free-flight section trajectory model}

The projectile free-flight section is basically in conformity with the trajectory of ordinary shrapnel and is under the influence of gravity, air resistance and polar damping torque. Considering the influence of longitudinal winds and cross winds, the tour degree-of-freedom movement differential equation of the projectile in the free-flight section is as the following: 


$$
\left\{\begin{array}{l}
\frac{d v_{x}}{d t}=-\frac{1}{2 m_{1}} \rho v_{r} C_{x} S_{1}\left(v_{x}-w_{x}\right) \\
\frac{d v_{y}}{d t}=\frac{1}{2 m_{1}} \rho v_{r} C_{x} S_{1} v_{y}-g \\
\frac{d v_{z}}{d t}=-\frac{1}{2 m_{1}} \rho v_{r} C_{x} S_{1}\left(v_{z}-w_{z}\right) \\
\frac{d \dot{r}}{d t}=-v_{r} k_{x r} \dot{r} \\
\frac{\mathrm{d} x}{\mathrm{~d} t}=v_{x} ; \frac{\mathrm{d} y}{\mathrm{~d} t}=v_{y} ; \frac{\mathrm{d} z}{\mathrm{~d} t}=v_{z}
\end{array}\right.
$$

In this section, such factors can be defined: the coordinates $\mathrm{p}(\mathrm{x}, \mathrm{y}, \mathrm{z})$, velocity $\left(\mathrm{v}_{\mathrm{x}}, \mathrm{v}_{\mathrm{y}}, \mathrm{v}_{\mathrm{z}}\right)$ and flight time t, the trajectory inclination angle and rotational speed of cabin-opening point.

\section{The building of scout equipment projecting section trajectory model}

During the projecting process, due to the constraint effect of the projectile bore, the scout equipment is only able to move along the projectile axis. Thus, the projectile axis coordinate system is chose to build the scout equipment projecting section trajectory model. In the projectile axis coordinate system $\mathrm{O}-\mathrm{X}_{1} \mathrm{Y}_{1} \mathrm{Z}_{1}$, the original point $\mathrm{O}$ is the mass center of the scout equipment, $\mathrm{OX}_{1}$ axis is in line with projectile axis and points to the direction of the velocity, $\mathrm{OY}_{1}$ axis is perpendicular to $\mathrm{OX}_{1}$ axis in the vertical plane, and the downward direction is the positive line, $\mathrm{OZ}_{1}$ axis is defined with the right hand rule.

During the projecting process, the external forces acting on the scout equipment includes: gravity, the thrust of powder gas, friction which caused by relative motion and inertial force in the non-inertial system of coordinates.

(1)The axial movement differential equation of the scout equipment

(1) Gravity

In the projectile axis coordinate system, three gravity components in the scout equipment are

$$
\left[\begin{array}{c}
\left(F_{g}\right)_{x_{1}} \\
\left(F_{g}\right)_{y_{1}} \\
\left(F_{g}\right)_{z_{1}}
\end{array}\right]=\left[\begin{array}{c}
-m g \sin \varphi_{\mathrm{a}} \cos \varphi_{2} \\
-m g\left(\cos \gamma \cos \varphi_{\mathrm{a}}-\sin \gamma \sin \varphi_{\mathrm{a}} \sin \varphi_{2}\right) \\
m g\left(\sin \gamma \cos \varphi_{\mathrm{a}}+\cos \gamma \sin \varphi_{\mathrm{a}} \sin \varphi_{2}\right)
\end{array}\right]
$$

(2)The thrust of powder gas

In the projectile axis coordinate system, the thrust of powder gas is

$$
\left[\begin{array}{l}
\left(F_{p}\right)_{x_{1}} \\
\left(F_{p}\right)_{y_{1}} \\
\left(F_{p}\right)_{z_{1}}
\end{array}\right]=\left[\begin{array}{c}
-S P \\
0 \\
0
\end{array}\right]
$$

(3)Inertial force

The relative velocity in the projectile body coordinate system is $v_{M_{r}}$, then the coriolis acceleration is:

$$
\overrightarrow{a_{k}}=2 \vec{\Omega} \times \vec{v}_{M_{r}}
$$

The coriolis acceleration in projectile axis coordinate system is:

$$
\left[\begin{array}{l}
\left(a_{k}\right)_{x_{1}} \\
\left(a_{k}\right)_{y_{1}} \\
\left(a_{k}\right)_{z_{1}}
\end{array}\right]=2\left[\begin{array}{ccc}
0 & -\Omega_{z_{1}} & \Omega_{y_{1}} \\
\Omega_{z_{1}} & 0 & -\Omega_{x_{1}} \\
-\Omega_{y_{1}} & \Omega_{x_{1}} & 0
\end{array}\right] \cdot\left[\begin{array}{l}
v_{M x_{1}} \\
v_{M y_{1}} \\
v_{M z_{1}}
\end{array}\right]=\left[\begin{array}{l}
2\left(\Omega_{y_{1}} v_{M z_{1}}-\Omega_{z_{1}} v_{M y_{1}}\right) \\
2\left(\Omega_{z_{1}} v_{M x_{1}}-\Omega_{x_{1}} v_{M z_{1}}\right) \\
2\left(\Omega_{x_{1}} v_{M y_{1}}-\Omega_{y_{1}} v_{M x_{1}}\right)
\end{array}\right]
$$

According to Newton's second law, the equation of motion of point $\mathrm{M}$ with mm mass is:

$$
m_{M} \mathrm{a}_{M}=\sum \mathrm{F}
$$


Because of the relative motion between scout equipment and bore, so it is

$$
\begin{gathered}
M \overrightarrow{\mathrm{a}}_{M_{r}}=\sum \overrightarrow{\mathrm{F}}-M \overrightarrow{\mathrm{a}}_{M_{e}}-M \overrightarrow{\mathrm{a}}_{k} \\
\overrightarrow{\mathrm{G}}_{M}=-M \overrightarrow{\mathrm{a}}_{M_{e}}-M \overrightarrow{\mathrm{a}}_{k}
\end{gathered}
$$

And

$$
M \vec{a}_{M_{r}}=\sum \overrightarrow{\mathrm{F}}+\overrightarrow{\mathrm{G}}_{M}
$$

Seen by the formula ( 8 ), inertia force $\vec{G}_{M}$ is constituted by implicated inertia force and coriolis inertia force.

The velocity of scout equipment is

$$
\left[\begin{array}{l}
\left(v_{r}\right)_{x_{1}} \\
\left(v_{r}\right)_{y_{1}} \\
\left(v_{r}\right)_{z_{1}}
\end{array}\right]=\left[\begin{array}{c}
-v_{d} \\
0 \\
0
\end{array}\right]
$$

Thus, the inertial forces in projectile axis coordinate system is:

$$
\left[\begin{array}{c}
G_{x_{1}} \\
G_{y_{1}} \\
G_{z_{1}}
\end{array}\right]=-m \cdot\left[\begin{array}{c}
a_{o x_{1}}-\Omega_{y_{1}}^{2} x-\Omega_{z_{1}}^{2} x \\
a_{o_{y_{1}}}+\dot{\Omega}_{z_{1}} x+\Omega_{x_{1}} \Omega_{y_{1}} x+2 \Omega_{z_{1}} v_{d} \\
a_{o_{z_{1}}}-\dot{\Omega}_{y_{1}} x+\Omega_{x_{1}} \Omega_{z_{1}} x-2 \Omega_{y_{1}} v_{d}
\end{array}\right]
$$

\section{(4)Friction}

Because the bomb around projectile axis coordinate with high-speed rotating, scout equipment snaps the bore with the centrifugal inertial force. When the scout equipment in the axial motion, there is axial friction, in the same time, the friction is produced by additional inertial forces cause swing and reconnaissance devices. Furthermore, the friction is produced by gravity.

The inertia force action on the bore of bomb, when the scout equipment moves, the friction is produced. The direction is different from movement of scout equipment. Set the friction coefficient is $\mu$, the friction of scout equipment is:

$$
R_{s}=\mu\left(G_{s}\right)_{\overline{o I}}
$$

In fact, besides the radial force, the horizontal component should also be decomposed into vertical and radial normal force without friction. It produces the reconnaissance device relative to the mother bore sliding trend, In general, considering the normal component cannot cause sliding reconnaissance device, it can omit.

Additional friction caused by gravity is:

$$
R_{g}=\mu m g\left[-\cos \gamma \cos \varphi_{a}+\sin \gamma \sin \varphi_{a} \sin \varphi_{1}+\sin \gamma \cos \varphi_{a}+\cos \gamma \sin \varphi_{a} \sin \varphi_{1}\right]
$$

So, the friction formula is:

$$
R=R_{s}+R_{g}
$$

(2) Differential equation of device for axial movement reconnaissance

Because of the mother chamber wall constraint, reconnaissance device movement in the axial coordinate systems is only the axial movement, the equation of motion vector device is:

$$
m \vec{a}_{r}=\sum \vec{F}+\vec{G}
$$

Put the projection of formula to the ${ } x_{1}$ axis:

$$
m \frac{\mathrm{d}\left(-v_{d}\right)}{\mathrm{d} t}=\left(F_{g}\right)_{x_{1}}+\left(F_{p}\right)_{X_{1}}+(G)_{x_{1}}+R
$$

When

the

reconnaissance equipment transported to the specified target region, burning propellant under the time fuze. The production of high-pressure gas pushing board, thrust through the housing, a semicircular plate, supporting the tile is transferred to the bottom, and the bottom shear elastic thread will reconnaissance device was thrown from the bomb, the ejection process, due to the constraint effect of bomb chamber, reconnaissance device can only play along the parent axis motion, force to reconnaissance unit the inertia force, gravity, friction powder gas thrust caused by the relative 
motion and non inertial coordinate system, reconnaissance device projection section along the projectile movement in the direction of axial elastic differential equation.

$$
\frac{\mathrm{d} v_{d}}{\mathrm{~d} t}=\frac{1}{m_{2}}\left(S_{2} P-R\right)+a_{o x_{1}}-\Omega_{y_{1}}^{2} x-\Omega_{\mathrm{z}_{1}}^{2} x+g \sin \varphi_{\mathrm{a}} \cos \varphi_{2}
$$

\section{Determine the reconnaissance device ejection velocity}

Reconnaissance device from a bomb thrown in the process, the thrust, gravity and friction, relative axial velocity $v_{\tau}$ and velocity $v_{n}$, the actual speed reconnaissance device can be obtained after projection conversion to ground coordinates through the elastic axis coordinate system, the right angle coordinate system $p-x y z, p$ in projectile point, xpy plane and shooting plumb surface coincidence, $x p z$ plane is the plane, let $v_{m}$ be a projectile points of the projectile velocity, $v_{p / l}$ is the axial ejection velocity reconnaissance device relative bombs, $v_{p \perp}$ As the radial ejection velocity reconnaissance device relative bombs, it is because the projectile rotation or ejection device wither reconnaissance device, the velocity vector is shown in picture 1.

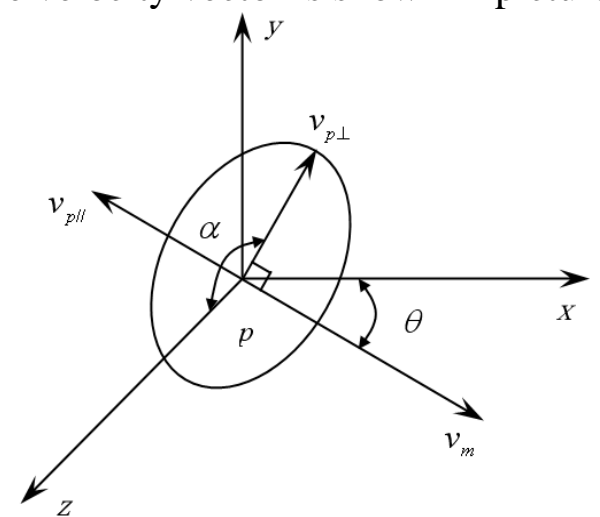

Fig.1 Velocity vectorgraph

When reconnaissance device throwing, original velocity are maked through putting $v_{m}, v_{p / \prime}$ and $v_{p \perp}$ to the axis of $p-x y z$.

$$
\left\{\begin{array}{l}
v_{x}=\left(v_{m}-v_{p / /}\right) \cos \theta-v_{p \perp} \sin \alpha \cos \theta \\
v_{y}=\left(v_{m}-v_{p / /}\right) \sin \theta+v_{p \perp} \sin \alpha \sin \theta \\
v_{z}=v_{p \perp} \cos \alpha
\end{array}\right.
$$

\section{Simulation and results analysis}

The effect of projectile velocity detection device, thereby changing reconnaissance device from slowdown to stable falling this process of flight time and flight distance, table1 table 3 are listed to effect projectile velocity, projecting loading and projecting height on the projectile velocity and projecting time.

Table 1 Effect of projectile velocity on projecting velocity and projecting time

\begin{tabular}{|c|c|c|c|c|c|}
\hline$\dot{r}_{0} /\left(\mathrm{r} \cdot \mathrm{s}^{-1}\right)$ & 1617.4 & 1695.3 & 1779.1 & 1857.4 & 1940.9 \\
\hline$v_{d} /\left(\mathrm{m} \cdot \mathrm{s}^{-1}\right)$ & 72.49 & 70.55 & 68.23 & 65.6 & 61.38 \\
\hline$t_{s} / \mathrm{ms}$ & 1.50 & 1.53 & 1.57 & 1.63 & 1.69 \\
\hline
\end{tabular}

From table 1, when changing projectile velocity, the impact is as follow.

Great influence on projectile velocity is projecting velocity and projecting time, when the velocity increases, projecting velocity decreases significantly, mainly due to the velocity increase, caused by the rapid increase of friction. 
Table 2 Effect of projecting charge on projecting velocity and projecting time

\begin{tabular}{|c|c|c|c|c|c|}
\hline$m_{\omega} / \mathrm{kg}$ & 0.0081 & 0.0086 & 0.0091 & 0.0096 & 0.0101 \\
\hline$v_{d} /\left(\mathrm{m} \cdot \mathrm{s}^{-1}\right)$ & 56.46 & 60.16 & 64.50 & 68.87 & 72.49 \\
\hline$t_{s} / \mathrm{ms}$ & 1.75 & 1.63 & 1.52 & 1.48 & 1.40 \\
\hline
\end{tabular}

From table 2, when changing projecting charge, the impact is as follow. reduces.

With increasing projecting charge, projecting velocity increases monotonically, projecting time

Table 3 Effect of projecting height on projecting velocity and projecting time

\begin{tabular}{|c|c|c|c|c|c|}
\hline$H / \mathrm{m}$ & 800 & 1000 & 1100 & 1200 & 1500 \\
\hline$v_{d} /\left(\mathrm{m} \cdot \mathrm{s}^{-1}\right)$ & 63.56 & 63.34 & 63.21 & 63.14 & 63.03 \\
\hline$t_{s} / \mathrm{ms}$ & 1.56 & 1.57 & 1.57 & 1.58 & 1.59 \\
\hline
\end{tabular}

From table 3, when changing projecting height, the impact is as follow.

With increasing projecting height, impact on the projecting velocity and projecting time is very small.

\section{Conclusion}

(1)In the selection of video scout projectile opening-point, the downward arc stage is chose. Because temporality angular velocity is relatively small in the air, reconnaissance device is thrown stability, thus increasing reconnaissance device working time, it can be on the target of long time effective reconnaissance.

(2)Because of projecting charge increasing, projecting velocity increased significantly, so in the design of video scout projectile, according to the projecting mechanism strength, reliability, appropriate projecting charge chose.

(3)Because of the effect of changing projecting height on projecting velocity and projecting time is very small, so according to the working conditions and operational requirements, projecting height is as far as possible to choose higher.

\section{References}

[1] Qian Lizhi. Suspension Technology and Application [M].Beijing: Peoples Liberation Army Publishing House. 2005:36-72

[2] Yang Qiren. Shrapnel Fight Dynamics [M].Beijing: National Defence of Industry Press, 1999. 82-140

[3] Li Dayao. Using Rigid Body-particle Trajectory Model Method to Calculate Projectileparachute System Basic Equations [J].Spacecraft Recovery \& Remote Sensing,1995,16(3):4-16

[4] Guo Rui etc. Dynamics Model for Terminal-Sensitive Submunition in Deceleration Stage [J]. Journal of Projectiles, Rockets, Missiles and Guidance,2005(4):186-189 